\title{
Innovative Grounding Methodology for Epoxy Impregnated Semiconductor Cross Sections for Electron Microscopy Inspection
}

\author{
Frieder Baumann, Pradip Sairam Pichumani and Christopher Torcedo
}

Globalfoundries, Inc., Malta, New York, United States

The semiconductor industry presents a challenging production scenario by pushing the technology envelope of integrated circuit packaging. The demand for shrinking the geometry and introducing cost competitive materials and processing technology pose a constant learning and improvement opportunity for failure analysis labs. Mechanical cross sectioning technique has been in use in failure analysis labs since the 1950s. This technique provides the analyst with a wealth of information about the IC device such as the critical dimensions of each layer, layer structures, grain growth of various crystals in the layers, along with existence and confirmation of defects. Recent advances in Focused Ion Beam systems and cross section polishers have pushed sample preparation towards artifact free cross sections [1]. However, mechanical cross section still remains a reliable method, especially for large area cross sections. Many defects including shorts, delamination, ESD related defects, solder cracking, and non-wet issues, which can only be verified physically in cross section.

One of the critical analysis obstacles for samples impregnated in epoxy is specimen charging during scanned electron beam imaging, which is caused by the difference between the numbers of primary and emitted electron species. In the case of epoxy and the non-conductive materials in the sample of interest, the dissipated charge does not flow off the electron scanning point fast enough. Samples in epoxy are often an island surrounded by resin. The electric field created by the localized charge on the surface influences the trajectories of both the primary and signal electrons moving towards the detector so that the geometry as well as the intensity scale of the image are distorted [2]. To address the charging issues, numerous treatments have been studied and presented by the failure analysis community. This paper focuses on the technique developed by the authors to counter the electric field generated at the surface by adding electrically conductive structures into the epoxy supporting the sample. Earlier iterations of the grounding structure by the authors included incorporation of metal pillars in epoxy making contact with sample. However, regardless of their dimension, distribution, or spacing, the mere presence of metal pillars would cause significant wear on the sandpaper and polishing pads. The mixing of conductive filler with epoxy caused the puck to lose its transparency, making it difficult to visually gauge the sample's polishing progress. The final solution proposed in this paper is the fabrication of the grounding structures in advance and then incorporating them into the conventional epoxy impregnation process along with the sample.

In this study, the authors compared conventionally prepared epoxy puck with the modified puck with grounding structures as shown in figure 1. Both the types were $\mathrm{Cr}$ coated prior to SEM imaging for a period of 100 seconds in order to establish the grounding structures as the only variable component in the study. Charging artifacts were not observed in the case of epoxy puck with grounding structures, while severe image distortion was observed in the case of conventional epoxy as shown in figure 2. Future work includes a slotted design in conventional epoxy puck to remove the cross sectioned sample upon completion of the polishing process [3]. 

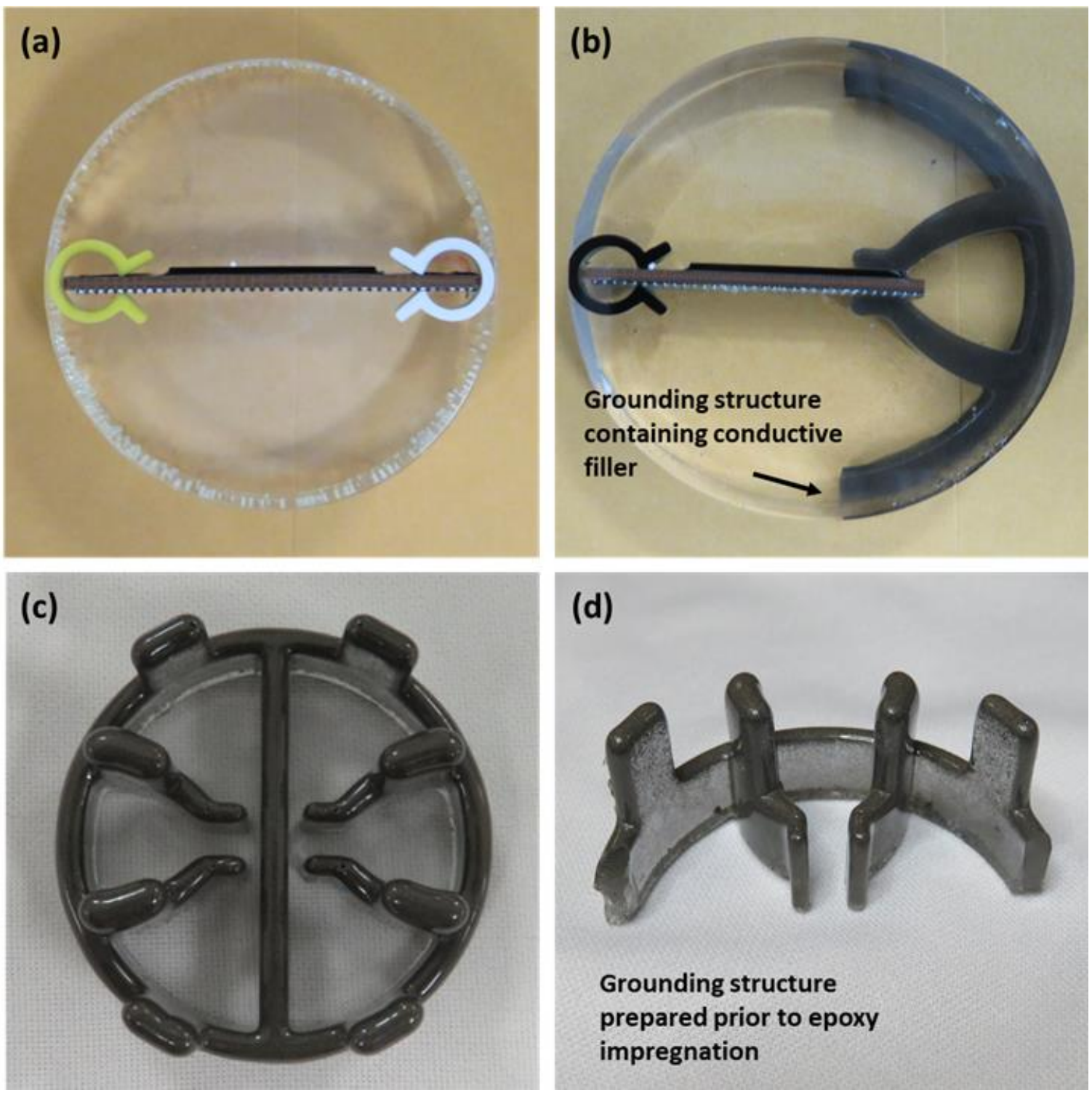

Figure 1. Optical image of (a) a conventional epoxy puck, (b) epoxy puck with grounding structure, (c) $\&$ (d) grounding structure prepared prior to epoxy impregnation of the sample 

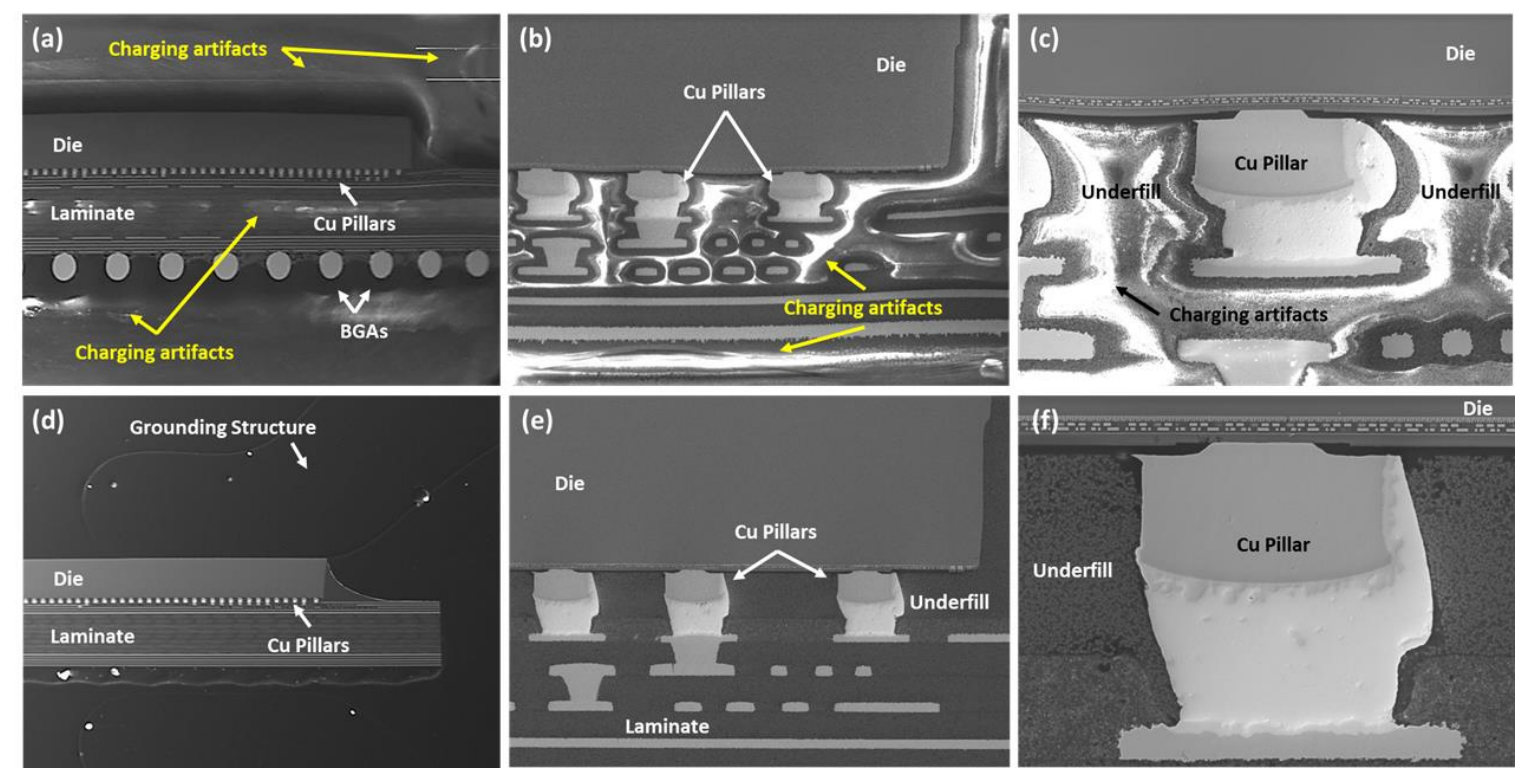

Figure 2. (a) Low magnification SEM image of the conventional epoxy puck. Charging artifacts were observed. (b) \& (c) are high magnification SEM images of the conventional epoxy puck cross section. Severe image distortion observed due to charging issues. Image (d) represents the SEM overview of the epoxy puck with the grounding structure. (e) \& (f) are high magnification SEM images of the epoxy puck with grounding structure. No charging related artifacts observed.

\section{References}

[1] F. Khatkhatay et al. A Simple Method to Decouple Redeposition-Related Artifacts from Real Defects in the Failure Analysis of Silicon Photonics Modules. M\&M 2019, 726-727.

[2] L. Frank, et al. Scanning Electron Microscopy of Nonconductive Specimens at Critical Energies in a Cathode Lens System. Scanning. 23. 36-50. 10.1002/sca.4950230106.

[3] The authors would like to thank Jeremy Brundige for the mechanical cross section and imaging 